\title{
CRESCIMENTO DE UM CLONE DE EUCALIPTO SUBMETIDO A DESRAMA E DESBASTE ${ }^{1}$
}

\author{
Marco Antonio Monte ${ }^{2}$, Maria das Graças Ferreira Reis ${ }^{3}$, Geraldo Gonçalves dos Reis ${ }^{3}$, Helio Garcia \\ Leite $^{3}$, Filipe Valadão Cacau ${ }^{4}$ e Frederico de Freitas Alves ${ }^{2}$
}

\begin{abstract}
RESUMO - Plantas de clone de Eucalyptus grandis, em região de Cerrado, no espaçamento inicial de 3 x 3 m, foram submetidas à desrama artificial e ao desbaste. Os tratamentos de desrama tiveram início em três idades diferentes: 16, 20 ou 28 meses. Foram utilizadas três a quatro intervenções de desrama, com remoção de copa em diferentes alturas, para atingir $6 \mathrm{~m}$ de fuste livre de nó, quando se iniciou a desrama aos 16 ou 20 meses, e duas a três intervenções, quando se iniciou aos 28 meses. Foram aplicados dois tratamentos de desbaste aos 55 meses, com remoção de 0 e $35 \%$ do número de mudas plantadas. O crescimento foi avaliado no período pós-desbaste, entre 55 e 93 meses de idade, por meio da análise do diâmetro (DAP), altura total (Ht), volume com casca $(V)$, incremento periódico anual pós-desbaste (IPA) e incremento médio anual (IMA). Aos 93 meses, não foram observadas diferenças significativas ( $>>0,05)$ em $D A P, H t, V, I P A$ e IMA, entre os tratamentos de desrama, e na interação entre desrama e desbaste. No tratamento desramado até 1,5 m de altura, aos 16 meses, houve remoção de $80 \%$ da biomassa de folhas e galhos. Nesse tratamento, $65 \%$ dos indivíduos estavam com $D A P$ inferior a $15 \mathrm{~cm}$, em comparação com $35 \%$ nos demais tratamentos. A $H t$ no povoamento desbastado foi menor $(\mathrm{p} \leq 0,05)$ do que no não desbastado. O $V$, o IPA e o IMA foram, respectivamente, de 7,9, 23,0 e $7,3 \%$ mais elevados $(\mathrm{p} \leq 0,05)$ no povoamento desbastado, quando a primeira intervenção de desrama foi aplicada aos 20 meses. O povoamento não desbastado teve crescimento estagnado aos 80 meses. No desbastado, essa estagnação deverá ocorrer entre 100 e 110 meses de idade, indicando necessidade de aplicação do segundo desbaste nessa faixa etária, para obtenção de toras de maior diâmetro e, consequentemente, maior extensão de madeira limpa.
\end{abstract}

Palavras-chave: Crescimento de plantas, desbaste e desrama artificial.

\section{GROWTH OF EUCALYPT CLONE SUBMITTED TO PRUNING AND THINNING}

\begin{abstract}
Plants of Eucalyptus grandis clone, established in a $3 \times 3 \mathrm{~m}$ spacing, were submitted to pruning and thinning. Pruning treatments started at three ages: 16, 20 or 28 months. Three or four pruning interventions were applied at different tree heights to attain a $6 \mathrm{~m}$ log free of branches, when pruning started at the age of 16 or 20 months, and two or three pruning were applied for the treatments starting at the age of 28 months. At the age of 55 months, two thinning treatments were applied (control and removal of $35 \%$ of the total planted seedlings). The plant growth after thinning was evaluated for the period between 55 to 93 months by analyzing diameter $(D B H)$, total height $(H t)$, volume outside bark $(V)$, periodic annual increment after thinning (PAI) and mean annual increment (MAI). At the age of 93 months, there were no significant differences $(p>0.05)$ for the diameter at $1.3 \mathrm{~m}$ height $(\mathrm{DBH})$, total height $(\mathrm{Ht})$, volume outside bark $(\mathrm{V})$, periodic annual increment after thinning (PAI) and mean annual increment (MAI) between artificial pruning treatments and for the pruning-thinning interaction. By pruning up to the height of $1.5 \mathrm{~m}$ in the first intervention, at the age of 16 months, there was a removal of $80 \%$ of total leaves and branches. In this treatment, $65 \%$ of the individuals presented diameter smaller than $15 \mathrm{~cm}$ in comparison to $35 \%$ for the remaining treatments.
\end{abstract}

\footnotetext{
${ }^{1}$ Recebido em 07-11-2007 e aceito para publicação em 23.06.2009.

${ }^{2}$ Programa de Pós-Graduação em Ciência Florestal da UFV. E-mail: <marco.monte@ufv.br> e <frederico.alves@ufv.br>.

${ }^{3}$ Departamento de Engenharia Florestal da Universidade Federal de Viçosa (UFV). E-mail: <mgfreis@ufv.br>, <greis@ufv.br> e<hgleite@ufv.br>.

${ }^{4}$ Plantar Reflorestamento, MS - Brasil. E-mail: <filipe_cacau@plantar.com.br>.
} 


\begin{abstract}
The total height was significantly $(p \leq 0.05)$ higher in the non-thinned stand in comparison to the thinned one. The volume outside bark, PAI and MAI varied significantly $(p \leq 0.05)$ with thinning only when pruning started at the age of 20 months, being, respectively, 7.9, 23.0 and $7.3 \%$ greater in the thinned stand, in comparison to the non-thinned one. It was observed that growth stagnated at the age of 80 months in the non-thinned stand. In the thinned stand, this stagnation may occurs around the age of 100-110 months, indicating that a second thinning should be applied by this age to obtain logs of larger diameter and, consequently, greater extension of clear wood.
\end{abstract}

Keywords: Plant growth, thinning and artificial pruning.

\section{INTRODUÇÃO}

A utilização da madeira de eucalipto deve-se às suas características físico-mecânicas e estéticas diferenciadas (PEREIRA et al., 2000), destacando o uso para produção de móveis. A possibilidade de uso de rotações curtas de povoamentos estabelecidos com espécies de rápido crescimento viabiliza o seu manejo para fins mais nobres, porém para produzir madeira com qualidade para fabricação de móveis é necessário aplicar desrama artificial.

A desrama artificial possibilita, principalmente, a obtenção de madeira serrada livre de nós (PIRES, 2000; VALE et al., 2002; ALMEIDA, 2003; POLLI et al., 2006). Porém, há necessidade de obter-se madeira de qualidade sem a redução na produção. Alguns estudos indicam que a desrama artificial não influencia significativamente o crescimento em diâmetro, altura e volume das árvores em povoamentos de eucalipto, quando o material genético tem capacidade de recompor sua área foliar, ou quando a remoção de área foliar é reduzida e constituída, principalmente, de folhas basais, que já se encontram em senescência (ALMEIDA, 2003; LIMA, 2003). Contudo, remoção drástica de ramos das árvores implica retirada de folhas da porção mediana da copa, com elevada capacidade fotossintética (BEADLE et al., 2007). Isso pode promover redução no crescimento das plantas (PIRES et al., 2002; PINKARD, 2003), devido à lenta recomposição de copa. Desse modo, a definição adequada da intensidade, da frequência e da idade das plantas, por ocasião da aplicação da desrama artificial, evita comprometer o crescimento, além de manter o núcleo nodoso reduzido, com consequente aumento da produção de madeira limpa (POLLI et al., 2006).

Em se tratando de produção de madeira serrada, além da necessidade de características adequadas, como reduzido número de nós, é importante obter árvores com fustes de maiores diâmetros, o que pode ser conseguido com a aplicação de desbaste (VALINGER et al., 2000; NOGUEIRA et al., 2001; DIAS et al., 2005; LEITE et al., 2005; NOGUEIRA et al., 2005; HEIKKILÄ et al., 2007) ou utilizando espaçamentos amplos (LELES et al., 2001).

Os efeitos da desrama e do desbaste no crescimento de plantas têm sido analisados de forma independente. Uma análise da interação entre essas duas práticas silviculturais foi realizada por Chaves (2005). Diante da carência de estudos, torna-se importante executar trabalhos que avaliem os benefícios da desrama e do desbaste, em conjunto, na produção de toras com elevada proporção de madeira limpa.

O objetivo deste estudo foi avaliar o crescimento de um povoamento de clone de Eucalyptus grandis submetido a diferentes intensidades e frequências de desrama artificial, associadas com desbaste, em Abaeté, Minas Gerais.

\section{MATERIAL E MÉTODOS}

Este estudo foi realizado em um povoamento de clone de Eucalyptus grandis W. Hill ex Maiden (clone 24504), estabelecido em novembro de 1998, sob espaçamento inicial de 3 × 3 m, no Município de Abaeté, MG, região de Cerrado, situado à latitude de $19^{\circ} 15^{\prime} 94^{\prime \prime} \mathrm{S}$, longitude de 45 44' $56^{\circ}$ "O e altitude de $647 \mathrm{~m}$, em um Latossolo Vermelho-Amarelo. A temperatura média anual é de $20,74^{\circ} \mathrm{C}$; a precipitação média anual, de $1.350 \mathrm{~mm}$; o déficit hídrico anual, de $142 \mathrm{~mm}$, entre abril e outubro; e a evapotranspiração potencial anual, de $1.216 \mathrm{~mm}$, referente ao período de 1999 a 2005.

Foram combinadas diferentes intensidades (altura de remoção dos galhos a partir do solo) e frequências (número de intervenções necessárias para atingir $6 \mathrm{~m}$ de fuste livre de galhos na planta) de desrama, formando

R. Árvore, Viçosa-MG, v.33, n.5, p.777-787, 2009

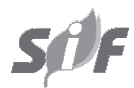


três conjuntos de tratamentos em função da idade em que se iniciou a desrama (16, 20 ou 28 meses). A testemunha foi comum para os três conjuntos de tratamentos de desrama.

Quando a desrama se iniciou aos 16 meses, foram aplicadas duas ou três intervenções até atingir a altura de $3 \mathrm{~m}$, sendo elas realizadas aos 16, 20 e 28 meses. Nos tratamentos de desrama com início aos 20 meses, também foram utilizadas duas ou três intervenções de desrama, porém, as idades de intervenções foram 20, 28 e 33 meses (Tabela 1). Em ambas as situações, foram aplicados os tratamentos: (T1) testemunha (plantas não desramadas); (T2) remoção de $0,5 \mathrm{~m}$ na primeira e segunda intervenção e 2,0 m na terceira; (T3) remoção de $0,5 \mathrm{~m}$ na primeira, $1,0 \mathrm{~m}$ na segunda e $1,5 \mathrm{~m}$ na terceira intervenção; (T4) remoção de 1,0 m em cada uma das três intervenções; (T5) remoção de 1,0 m na primeira e 2,0 m na terceira intervenção; e (T6) remoção de $1,5 \mathrm{~m}$ na primeira e $1,5 \mathrm{~m}$ na terceira intervenção. Nos tratamentos em que a primeira intervenção ocorreu aos 28 meses, foram feitas três desramas até atingir a altura de $3 \mathrm{~m}$. Essa idade foi utilizada em razão de ser comum e iniciar a desrama após a idade de 2 anos. Uma vez que nesta idade já havia ocorrido morte intensa de galhos da base das árvores e elevada senescência foliar até alturas superiores a $3 \mathrm{~m}$, a remoção de $0,5 \mathrm{~m}$ não foi realizada. Os tratamentos aplicados foram: (T7) remoção de 1,0 m na primeira (28 meses) e 2,0 m na segunda intervenção (33 meses); (T8) remoção de 1,5m na primeira e na segunda intervenção ( 28 e 33 meses) e (T9) remoção de 3,0 m em uma única intervenção, aos 28 meses de idade (Tabela 2). Na Tabela 3 são apresentados os dados de percentagem de biomassa de folhas e galhos removidos na primeira intervenção de desrama.

Aos 45 meses de idade, foi feita a intervenção de desrama em todos os tratamentos, removendo-se os galhos vivos e mortos entre 3 e $6 \mathrm{~m}$ de altura, para obtenção de uma segunda tora de $3 \mathrm{~m}$ livre de nós (Tabelas 1 e 2).

As parcelas foram constituídas de quatro fileiras, com 20 plantas cada. Aárea da parcela útil foi de $180 \mathrm{~m}^{2}$ e constituiu de 20 plantas, distribuídas nas duas linhas centrais (10 plantas em cada linha), a partir da quinta planta.

Tabela 1 - Intensidade e frequência da desrama aplicada em plantas do clone 24504 de E. grandis, com a primeira intervenção realizada aos 16 ou 20 meses de idade, em Abaeté, MG.

Table 1 - Pruning intensity and frequency treatments applied to the clone 24504 of $\boldsymbol{E}$. grandis starting at the age of 16 or 20 months, in Abaeté, MG, Brazil.

\begin{tabular}{|c|c|c|c|c|c|}
\hline \multirow[t]{2}{*}{ Tratamentos } & \multicolumn{4}{|c|}{ Altura parcial de desrama $(\mathrm{m})$} & \multirow[t]{2}{*}{ Altura total de desrama (m) } \\
\hline & $16^{1}$ ou $20^{2}$ meses & $20^{1}$ ou $28^{2}$ meses & $28^{1}$ ou $33^{2}$ meses & 45 meses & \\
\hline $\mathrm{T} 1$ & - & - & - & - & - \\
\hline $\mathrm{T} 2$ & $0,5^{3}$ & $0,5^{4}$ & $2,0^{4}$ & $3,0^{4}$ & 6,0 \\
\hline T 3 & 0,5 & 1,0 & 1,5 & 3,0 & 6,0 \\
\hline $\mathrm{T} 4$ & 1,0 & 1,0 & 1,0 & 3,0 & 6,0 \\
\hline T 5 & 1,0 & - & 2,0 & 3,0 & 6,0 \\
\hline T 6 & 1,5 & - & 1,5 & 3,0 & 6,0 \\
\hline
\end{tabular}

${ }^{1}$ Idades em que houve desrama nos tratamentos cuja a primeira intervenção ocorreu aos 16 meses de idade. ${ }^{2}$ Idades em que houve desrama nos tratamentos cuja primeira intervenção ocorreu aos 20 meses de idade. ${ }^{3}$ Altura de desrama a partir do nível do solo; ${ }^{4}$ altura de desrama a partir da primeira, segunda e, ou, terceira intervenções de desrama.

Tabela 2 - Intensidade e frequência da desrama aplicada em plantas do clone 24504 de E. grandis, com a primeira intervenção realizada aos 28 meses de idade, em Abaeté, MG.

Table 2 - Pruning intensity and frequency treatments applied to the clone 24504 of $\boldsymbol{E}$. grandis starting at the age of 28 months, in Abaeté, MG, Brazil.

\begin{tabular}{|c|c|c|c|c|}
\hline \multirow[t]{2}{*}{ Tratamentos } & \multicolumn{3}{|c|}{ Altura parcial de desrama (m) } & \multirow[t]{2}{*}{ Altura total de desrama (m) } \\
\hline & 28 meses & 33 meses & 45 meses & \\
\hline T1 & $\bar{C}$ & $\bar{C}$ & $\bar{C}$ & $\bar{C}$ \\
\hline T7 & $1,0^{1}$ & $2,0^{2}$ & $3,0^{2}$ & 6,0 \\
\hline T 8 & 1,5 & 1,5 & 3,0 & 6,0 \\
\hline Т9 & 3,0 & 0,0 & 3,0 & 6,0 \\
\hline
\end{tabular}

${ }^{1}$ Altura de desrama a partir do nível do solo; ${ }^{2}$ altura de desrama a partir da primeira e, ou, segunda intervenções de desrama. 
Tabela 3 - Porcentagem de remoção de biomassa de folhas e galhos na primeira intervenção de desrama artificial realizada aos 16, 20 ou 28 meses de idade, em plantas do clone 24504 de Eucalyptus grandis, em Abaeté, MG.

Table 3 - Leaf and branch biomass removal (\%) in the first pruning intervention at the age of 16, 20 or 28 months, of E. grandis clone, in Abaeté, MG, Brazil.

\begin{tabular}{|c|c|c|c|c|c|c|}
\hline \multirow[t]{3}{*}{ Tratamentos } & \multicolumn{6}{|c|}{ Idade da primeira intervenção de desrama (meses) } \\
\hline & \multicolumn{2}{|c|}{16} & \multicolumn{2}{|c|}{20} & \multicolumn{2}{|c|}{28} \\
\hline & Folhas & Galhos & Folhas & Galhos & Folhas & Galhos \\
\hline T 1 & - & - & - & - & - & - \\
\hline $\mathrm{T} 2$ & 23 & 30 & 6 & 12 & - & - \\
\hline T 3 & 25 & 28 & 8 & 18 & - & - \\
\hline T 4 & 41 & 26 & 38 & 52 & - & - \\
\hline T 5 & 57 & 58 & 34 & 45 & - & - \\
\hline Т 6 & 80 & 80 & 49 & 64 & - & - \\
\hline T 7 & - & - & - & - & 10 & 14 \\
\hline T 8 & - & - & - & - & 18 & 24 \\
\hline Т 9 & - & - & - & - & 36 & 34 \\
\hline
\end{tabular}

Fonte: Lima (2000).

Aos 55 meses de idade, foi realizado o desbaste seletivo em toda a área das parcelas (incluindo bordadura), com redução de $35 \%$ no número de árvores (aproximadamente $27 \%$ da área basal do povoamento), sendo eliminadas árvores tortas, injuriadas, bifurcadas ou de menor porte em relação às demais, procurando manter certa uniformidade no espaço de crescimento (CHAVES et al., 2007).

O delineamento foi inteiramente casualizado, seguindo-se o esquema fatorial $6 \times 2$, nos tratamentos cuja desrama iniciou aos 16 ou 20 meses; e 4 x 2 nos tratamentos que tiveram desrama iniciada aos 28 meses, com três repetições.

No povoamento não desbastado foram marcadas, no campo, as árvores que poderiam ter sido eliminadas em um primeiro desbaste, correspondendo a $35 \%$ do número total de mudas plantadas. Essas árvores foram excluídas da análise, a fim de permitir a avaliação do crescimento somente das árvores que deveriam permanecer no povoamento, caso tivesse ocorrido o desbaste.

O crescimento das plantas foi avaliado por meio de medições do diâmetro a 1,3 m de altura (DAP) e da altura total (Ht), aos 55, 68, 81 e 93 meses de idade. Dados da cubagem de árvores obtidos nas idades de 55 e 93 meses de idade, foram utilizados para ajuste do modelo de Schumacher e Hall (1933) para estimar o volume com casca (V), em $\mathrm{m}^{3} \cdot \mathrm{ha}^{-1}$. Foram estimados, também, o incremento periódico anual (IPA) entre 55 e 93 meses e o médio anual (IMA).

Os dados de DAP, Ht, V, IPA e IMA foram comparados aos 93 meses, por meio da análise de variância (teste F) e do teste t de Wilcoxon (quando as pressuposições da Anova não foram atendidas). A função Weibull foi ajustada aos dados de frequência de árvores em classes diamétricas, para cada tratamento de desrama, nessa mesma ocasião. O modelo $L n V=\beta_{0}+\beta_{l} I^{-1}+\varepsilon$, em que $V=$ volume com casca $\left(\mathrm{m}^{3} \cdot \mathrm{ha}^{-1}\right)$ e $I=$ idade, foi ajustado em cada tratamento e as equações estimadas, comparadas por meio de testes de identidades de modelos $(\mathrm{p}=0,05)$.

\section{RESULTADOS E DISCUSSÃO}

O crescimento em altura e em DAP, bem como o IPA e o IMA, nos tratamentos cuja desrama iniciou aos 16 ou 20 meses, foram comparados pelo teste $F$, enquanto nos tratamentos com a primeira intervenção de desrama aos 28 meses essas variáveis foram analisadas pelo teste t de Wilcoxon, em razão de não ter atendido às pressuposições da análise de variância. Os volumes $\left(\mathrm{m}^{3} \cdot \mathrm{ha}^{-1}\right)$ foram comparados pelo teste de Wilcoxon em todos os tratamentos, independentemente da idade de início da desrama.

Aos 93 meses de idade, não foram observadas diferenças significativas ( $p>0,05)$ no crescimento em altura, entre os tratamentos de desrama e na interação desrama-desbaste, independentemente da idade de início da desrama (16, 20 ou 28 meses) (Tabela 4). Nessa idade, houve diferença $(\mathrm{p} \leq 0,05)$ na altura das árvores entre os tratamentos de desbaste. A altura média no povoamento desbastado foi 7,2\%,3,9\% e $10 \%$ menor do que a das árvores dominantes e codominantes no povoamento não desbastado (correspondentes a $65 \%$ das árvores plantadas), para as plantas cuja desrama iniciou aos 16, 20 ou 28 meses de idade, respectivamente (Tabela 4). Se todas as árvores do povoamento não 
desbastado tivessem sido consideradas na análise, provavelmente não seriam observadas diferenças significativas entre os tratamentos de desbaste. De modo geral, o crescimento em altura não é afetado significativamente pelo desbaste (MEDHURST et al., 2001; SULLIVAN et al., 2006; KANNINEN et al., 2004). Trevisan et al. (2007) não observaram diferenças entre alturas de E. grandis no espaçamento de 3,0 x 1,7 m, quando removeu $30 \%$ da área basal, mas a remoção de área basal superior a $60 \%$ favoreceu o crescimento em altura das árvores remanescentes.

O maior desenvolvimento em altura das árvores superiores no povoamento não desbastado, conforme observado no presente experimento, provavelmente ocorreu devido ao fato de haver maior competição entre plantas em razão de maior densidade, principalmente considerando que o clone estudado foi proveniente de Eucalyptus grandis, espécie altamente exigente em luz. É provável que em idades mais avançadas haja maior crescimento em altura no povoamento desbastado, pois, imediatamente após o desbaste, há maior alocação de carboidratos para a recomposição da copa, que está mais exposta à radiação, em detrimento do crescimento em altura. Chaves et al. (2007) observaram, nesse mesmo experimento, que houve redução significativa no índice de área foliar (IAF) do povoamento imediatamente após a aplicação do desbaste, que passou de 2,75 para 1,98, sendo que, seis meses após o desbaste, o IAF atingiu valores iguais ou superiores aos observados antes do desbaste, e 13 meses após o desbaste, a média geral do IAF chegou a 3,11. Medhurst et al. (2001) observaram em Eucalyptus nitens, plantado no espaçamento de 3,5 x 2,5 m, que a redução de $14 \%$ a $72 \%$ da área basal antes do desbaste não interferiu no crescimento em altura, porém houve aumento na razão média de copa viva das plantas remanescentes.

Tabela 4 - Diâmetro a 1,3 m de altura (DAP), altura total (Ht), volume com casca por hectare $\left(\mathrm{m}^{3}\right.$.ha $\left.\mathrm{a}^{-1}\right)$, incremento periódico anual pós-desbaste (IPA) e incremento médio anual (IMA), aos 93 meses de idade, em povoamento do clone 24504 de E. grandis submetido à desrama e ao desbaste, em Abaeté-MG.

Table 4 -Diameter at $1.3 \mathrm{~m}$ of height, total height, volume outside bark, periodic annual increment after thinning and mean annual increment at the age of 93 months, of $\boldsymbol{E}$. grandis clone submitted to artificial pruning and thinning, in Abaeté, MG, Brazil.

\begin{tabular}{|c|c|c|c|c|c|c|c|c|c|c|}
\hline \multirow[t]{2}{*}{ Tratamentos } & \multicolumn{5}{|c|}{ Desbastado } & \multicolumn{5}{|c|}{ Não desbastado ${ }^{1}$} \\
\hline & $\begin{array}{l}\text { DAP } \\
(\mathrm{cm})\end{array}$ & $\begin{array}{l}\mathrm{HT} \\
(\mathrm{m})\end{array}$ & $\begin{array}{l}\text { Volume } \\
\left(\mathrm{m}^{3} \cdot \mathrm{ha}^{-1}\right)\end{array}$ & \multicolumn{2}{|c|}{$\begin{array}{ll}\text { IPA }^{2} \text { IMA } \\
\left(\mathrm{m}^{3} \cdot \mathrm{ha}^{-1} \cdot \text { ano }^{-1}\right) \\
\end{array}$} & $\begin{array}{l}\text { DAP } \\
(\mathrm{cm})\end{array}$ & $\begin{array}{l}\mathrm{HT} \\
(\mathrm{m})\end{array}$ & $\begin{array}{l}\text { Volume } \\
\left(\mathrm{m}^{3} \cdot \mathrm{ha}^{-1}\right)\end{array}$ & \multicolumn{2}{|c|}{$\frac{\mathrm{IPA}^{2} \quad \mathrm{IMA}}{\left(\mathrm{m}^{3} \cdot \mathrm{ha}^{-1} \cdot \text { ano }^{-1}\right)}$} \\
\hline \multicolumn{11}{|c|}{ Primeira intervenção aos 16 meses } \\
\hline $\mathrm{T} 1$ & 15,6 & 22,3 & 119,5 & 18,4 & 19,2 & 16,1 & 24,0 & 131,5 & 20,1 & 17,0 \\
\hline $\mathrm{T} 2$ & 15,9 & 22,4 & 122,6 & 19,6 & 18,4 & 15,0 & 24,7 & 116,0 & 16,8 & 15,0 \\
\hline T3 & 15,8 & 23,5 & 132,7 & 22,5 & 19,7 & 15,5 & 24,6 & 124,5 & 18,9 & 16,1 \\
\hline $\mathrm{T} 4$ & 16,0 & 24,0 & 132,9 & 24,0 & 19,8 & 14,7 & 23,9 & 110,5 & 16,4 & 14,3 \\
\hline T 5 & 15,6 & 22,9 & 127,2 & 22,3 & 19,7 & 16,4 & 25,1 & 141,4 & 20,3 & 18,2 \\
\hline T 6 & 14,8 & 22,5 & 116,4 & 19,6 & 17,0 & 16,2 & 25,9 & 142,4 & 23,0 & 18,4 \\
\hline Média & 15,6 & 22,9 & 125,2 & 21,1 & 19,0 & 15,6 & 24,7 & 127,7 & 19,3 & 16,5 \\
\hline \multicolumn{11}{|c|}{ Primeira intervenção aos 20 meses } \\
\hline $\mathrm{T} 1$ & 15,6 & 22,3 & 119,5 & 18,4 & 15,4 & 16,1 & 24,0 & 131,5 & 20,1 & 17,0 \\
\hline $\mathrm{T} 2$ & 15,6 & 22,9 & 129,3 & 20,6 & 16,7 & 15,1 & 23,8 & 115,9 & 16,0 & 15,0 \\
\hline T3 & 15,3 & 22,8 & 125,8 & 20,5 & 16,2 & 15,0 & 23,6 & 107,3 & 14,3 & 13,8 \\
\hline $\mathrm{T} 4$ & 15,5 & 22,4 & 122,6 & 18,8 & 15,8 & 14,8 & 23,1 & 110,3 & 15,7 & 14,2 \\
\hline T 5 & 15,8 & 23,4 & 128,2 & 21,6 & 16,5 & 15,1 & 23,4 & 115,1 & 15,2 & 14,8 \\
\hline Т 6 & 15,2 & 23,0 & 126,0 & 20,8 & 16,3 & 15,0 & 24,5 & 116,5 & 16,8 & 15,0 \\
\hline Média & 15,5 & 22,8 & 125,2 & 20,1 & 16,2 & 15,2 & 23,7 & 116,1 & 16,3 & $\overline{15,0}$ \\
\hline \multicolumn{11}{|c|}{ Primeira intervenção aos 28 meses } \\
\hline $\mathrm{T} 1$ & 15,6 & 22,3 & 119,5 & 18,4 & 15,43 & 16,1 & 24,0 & 131,5 & 20,1 & 17,0 \\
\hline $\mathrm{T} 7$ & 14,7 & 21,0 & 103,8 & 17,1 & 13,39 & 15,3 & 24,0 & 118,9 & 16,3 & 15,3 \\
\hline $\mathrm{T} 8$ & 15,1 & 21,1 & 114,7 & 19,0 & 14,80 & 15,2 & 23,5 & 116,4 & 16,3 & 15,0 \\
\hline T9 & 14,8 & 21,1 & 111,9 & 18,1 & 14,45 & 14,6 & 23,6 & 108,1 & 15,3 & 13,9 \\
\hline Média & 15,1 & 21,4 & 112,5 & 18,2 & 14,51 & 15,3 & 23,8 & 118,7 & 17,0 & 15,3 \\
\hline
\end{tabular}

${ }^{1}$ Foram consideradas apenas as árvores que ficariam no povoamento caso tivesse sido realizado o primeiro desbaste.

${ }^{2}$ Refere-se ao incremento periódico anual após a realização do desbaste (55 meses), até aos 93 meses de idade. 

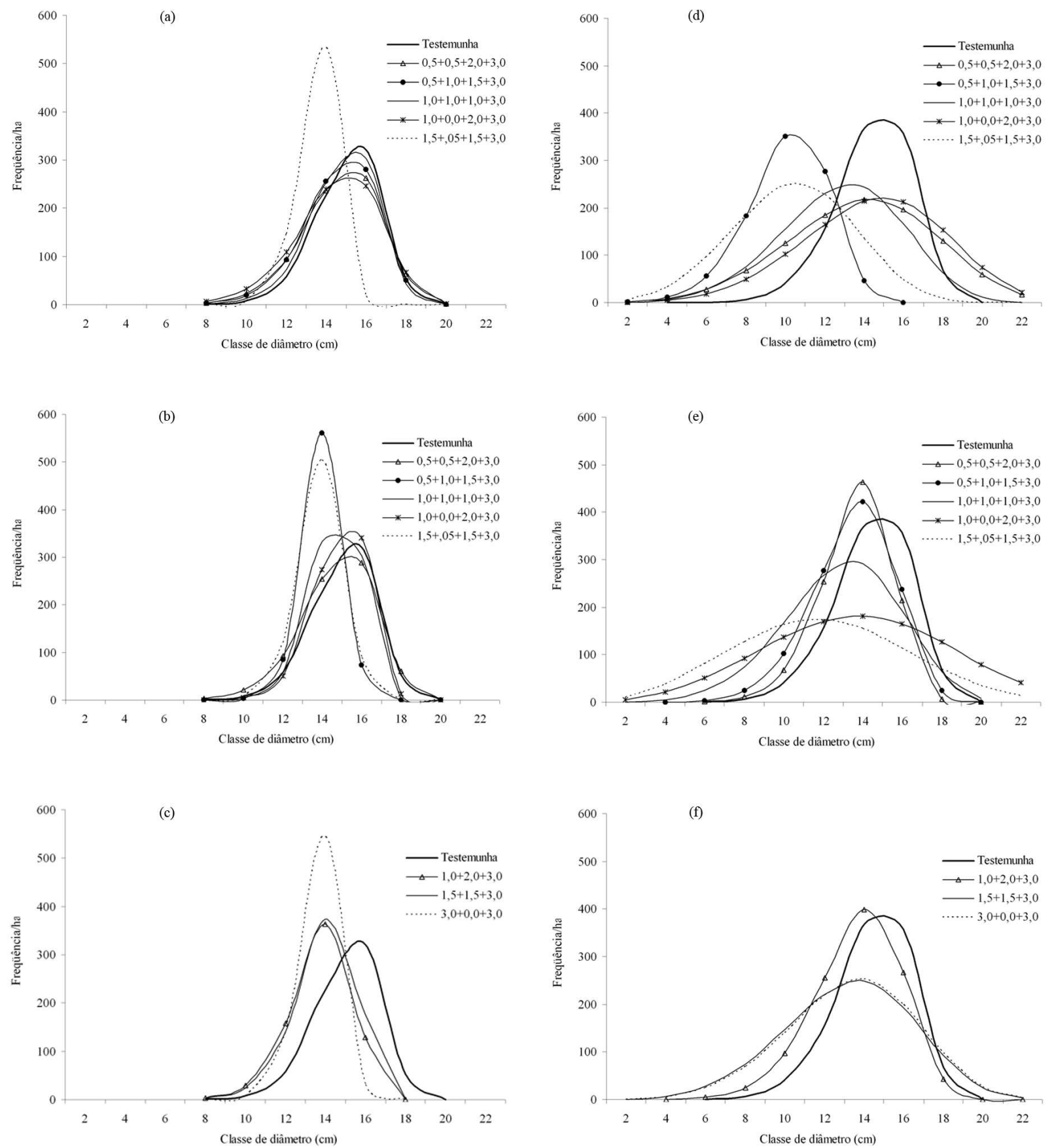

Figura 1 - Frequência de árvores por hectare, aos 93 meses de idade, obtida através da distribuição de Weibull nos tratamentos de desrama artificial iniciados aos 16 (a, d), 20 (b, e) ou 28 (c, f) meses de idade, em povoamentos desbastado (a, b, c) e não desbastado (c, d, f), do clone 24504 de E. grandis, em Abaeté-MG.

Figure 1 - Frequency of trees per hectare estimated using the Weibull distribution at age of 93 months, for each artificial pruning treatments, starting at the age of $16(a, d), 20(b, e)$ or $28(c, f)$ months, in thinned $(a, b, c)$ and nonthinned stands $(c, d, f)$ of an $\boldsymbol{E}$. grandis clone, in Abaeté, MG, Brazil.

R. Árvore, Viçosa-MG, v.33, n.5, p.777-787, 2009 
O DAP aos 93 meses não apresentou diferenças significativas $(p>0,05)$ entre os tratamentos de desrama e de desbaste e na interação desrama-desbaste, independentemente da idade de início da desrama (16, 20 ou 28 meses) (Tabela 4). Embora não tenham sido observadas diferenças significativas $(p>0,05)$ no DAP entre os tratamentos de desrama, verificou-se que a distribuição diamétrica segundo Weibull (Figura1) variou com esses tratamentos. Destaca-se o tratamento 6 (remoção de galho até $1,5 \mathrm{~m}$ de altura) em que a primeira intervenção de desrama ocorre aos 16 messes (Figura 1a), por ter apresentado $65 \%$ dos indivíduos com DAP inferior a $15 \mathrm{~cm}$, enquanto nos demais tratamentos, inclusive no testemunha, apenas $35 \%$ das plantas tiveram DAP inferior a $15 \mathrm{~cm}$, no povoamento desbastado. Nesse tratamento de desrama houve remoção de $80 \%$ de biomassa de folhas na primeira intervenção de desrama (Tabela 3), o que é considerado elevado. Ou seja, remoções drásticas de desrama podem comprometer o crescimento radial, uma vez que a planta tende, primeiramente, a recompor sua área foliar, conforme discutido por Lima (2003).

O desbaste de $35 \%$ das mudas plantadas adotado neste experimento não afetou, significativamente, o crescimento em diâmetro até três anos após essa intervenção. Kanninen et al. (2004) observaram que, com o desbaste leve ( $25 \%$ do número de árvores aos 4 ou 6 anos), não houve influência no crescimento em DAP das árvores de Tectona grandis, mas foi verificado maior DAP no povoamento submetido ao desbaste moderado $(25+25 \%$ no quarto e quinto anos) ou alto (60\% no quarto ano), em comparação com o povoamento não desbastado. Assim, intensidades de desbaste mais leves podem não afetar o crescimento das árvores, conforme observado por Dias et al. (2005) e, também, neste estudo. O efeito do desbaste sobre o crescimento da planta depende, ainda, da capacidade produtiva e da época de aplicações desse manejo (CAMPOS e LEITE, 2006). Maior crescimento em diâmetro pode ocorrer após a aplicação de um segundo desbaste.

O volume $\left(\mathrm{m}^{3} \cdot \mathrm{ha}^{-1}\right)$, o IPA e o IMA não apresentaram diferenças significativas ( $p>0,05)$ três anos após o desbaste, entre os tratamentos de desrama e de desbaste e na interação desrama-desbaste, quando a primeira intervenção de desrama ocorreu aos 16 ou 28 meses. Contudo, houve diferença significativa $(\mathrm{p} \leq 0,05)$ entre os tratamentos de desbaste, quando a primeira intervenção de desrama ocorreu aos 20 meses. Nesse caso, o volume, o IPA e o IMA foram, respectivamente, 7,9\%,23,0\% e 7,3\% mais elevados no povoamento desbastado (Tabela 4). Essa diferença indica que as árvores responderam positivamente à aplicação de desbaste, apesar de não terem sido adubadas após o desbaste. Medhurst et al. (2001) observaram maior crescimento das árvores dominantes e codominantes em resposta a aplicação do desbaste. Também, estes autores constataram que o incremento em área basal nos tratamentos desbastados foi sempre maior que no não desbastado. Kanninen et al. (2004) constataram que o volume no povoamento não desbastado, ou com desbaste leve, foi maior do que com desbaste moderado ou alto, quatro anos após a aplicação de desbaste, considerando todas as árvores do povoamento na avaliação. Nesta pesquisa, bem como no trabalho de Medhurst et al. (2001), foram consideradas apenas as árvores superiores do povoamento, em razão de apresentarem resultados contrastantes em relação ao observado por Kanninen et al. (2004). Trevisan et al. (2007) observaram, em E. grandis, no espaçamento de $3,0 \times 1,7$ m, que a remoção de $30 \%$ da área basal não interferiu na produção volumétrica das árvores remanescentes. Contudo, ao remover mais de $60 \%$ de área basal houve maior volume no povoamento desbastado.

Não houve diferença estatística $(p>0,05)$ entre as equações de produção estimadas para o povoamento desbastado nas três idades de primeira intervenção. Assim, uma equação comum foi estimada nos tratamentos de desrama artificial, dentro de cada grupo de idade de primeira intervenção de desrama (16, 20 ou 28 meses de idade), resultando, respectivamente, em: $\operatorname{Ln}(\mathrm{V})=5,94958-103,40005\left(\mathrm{I}^{-1}\right), \operatorname{Ln}(\mathrm{V})=5,85362$ $96,49122\left(\mathrm{I}^{-1}\right)$ e $\operatorname{Ln}(\mathrm{V})=5,76925-98,51465\left(\mathrm{I}^{-1}\right)$ (Figura 2).

No povoamento não desbastado, apenas as equações obtidas nos tratamentos de desrama iniciados aos 20 meses não foram diferentes entre si ( $p>0,05)$, pelo teste $\mathrm{F}$, sendo estimada a equação comum $\mathrm{Ln}(\mathrm{V})$ $=5,78683-72,25628\left(\mathrm{I}^{-1}\right)$ (Figura 3$)$. Quando a desrama se iniciou aos 16 meses de idade, o T4 diferiu dos demais tratamentos. Nesse caso, os tratamentos T1, T2, T3, T5 e T6, por terem sido estatisticamente iguais ( $p>0,05$ ), pelo teste $F$, foram representados por apenas uma equação comum, resultando em $\operatorname{Ln}(\mathrm{V})=5,92053$ 80,37815( $\left(\mathrm{I}^{-1}\right)$ (Figura 3). Quando a desrama se iniciou aos 28 meses de idade, os tratamentos T1 e T7 apresentaram-se iguais e foram representados por uma equação comum $\operatorname{Ln}(\mathrm{V})=5,96428-80,24904\left(\mathrm{I}^{-1}\right)$,

R. Árvore, Viçosa-MG, v.33, n.5, p.777-787, 2009 

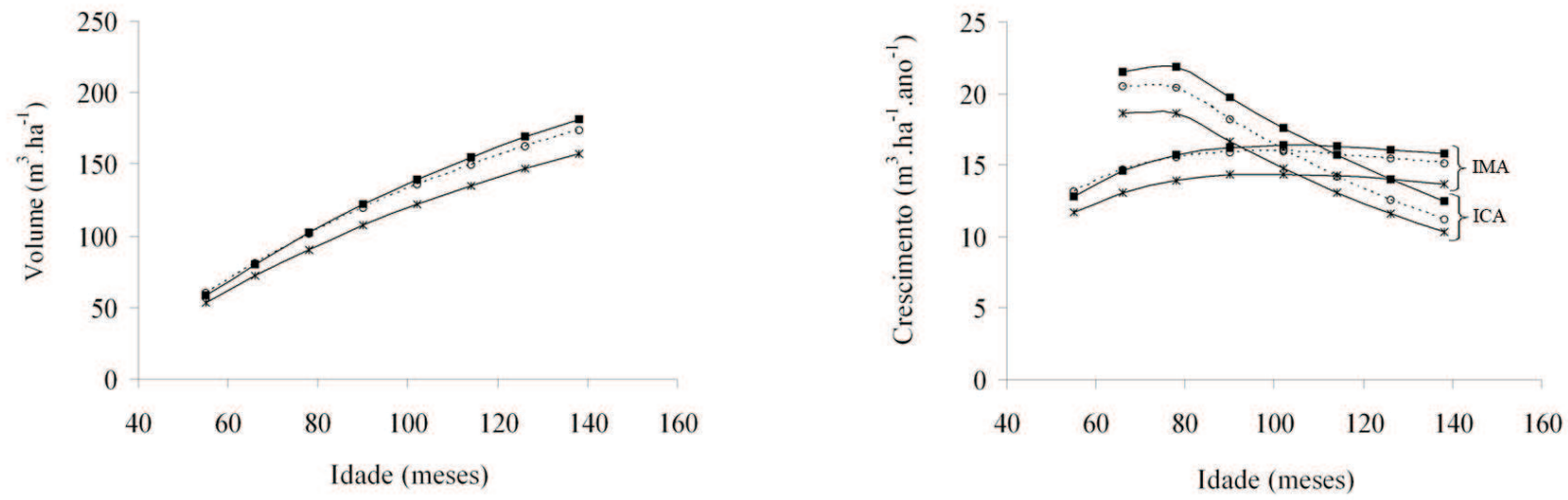

$$
-16 \text { meses }^{1} \quad-20 \text { meses }^{2} \quad * 28 \text { meses }^{3}
$$

Figura 2 - Volume $\left(\mathrm{m}^{3} \cdot \mathrm{ha}^{-1}\right)$, incremento médio anual $\left(\mathrm{m}^{3} \cdot\right.$ ha $^{-1} \cdot$ ano $\left.^{-1}\right)$ e incremento corrente anual $\left(\mathrm{m}^{3} \cdot \mathrm{ha}^{-1}\right.$. ano $\left.\mathrm{o}^{-1}\right)$ em povoamento do clone 24504 de E. grandis submetido ao desbaste e à desrama artificial, em Abaeté, MG. (16, 20 ou 28 meses = idades de primeira intervenção de desrama; 16 meses $^{1}=\mathrm{T} 1$ a T6; 20 meses $^{2}=\mathrm{T} 1$ a T6; e 28 meses $^{3}=\mathrm{T} 1$, T7, T8 e T9).

Figure 2 -Volume $\left(m^{3} \cdot h a^{-1}\right)$, mean annual increment $\left(m^{3} . h a^{-1} \cdot y r^{-1}\right)$, and periodic annual increment $\left(m^{3} . h a^{-1} . y r^{-1}\right)$, in a stand of $\boldsymbol{E}$. grandis clone submitted to artificial pruning (starting at the age of 16, 20 or 28 months) and thinning, in Abaeté, MG, Brazil.
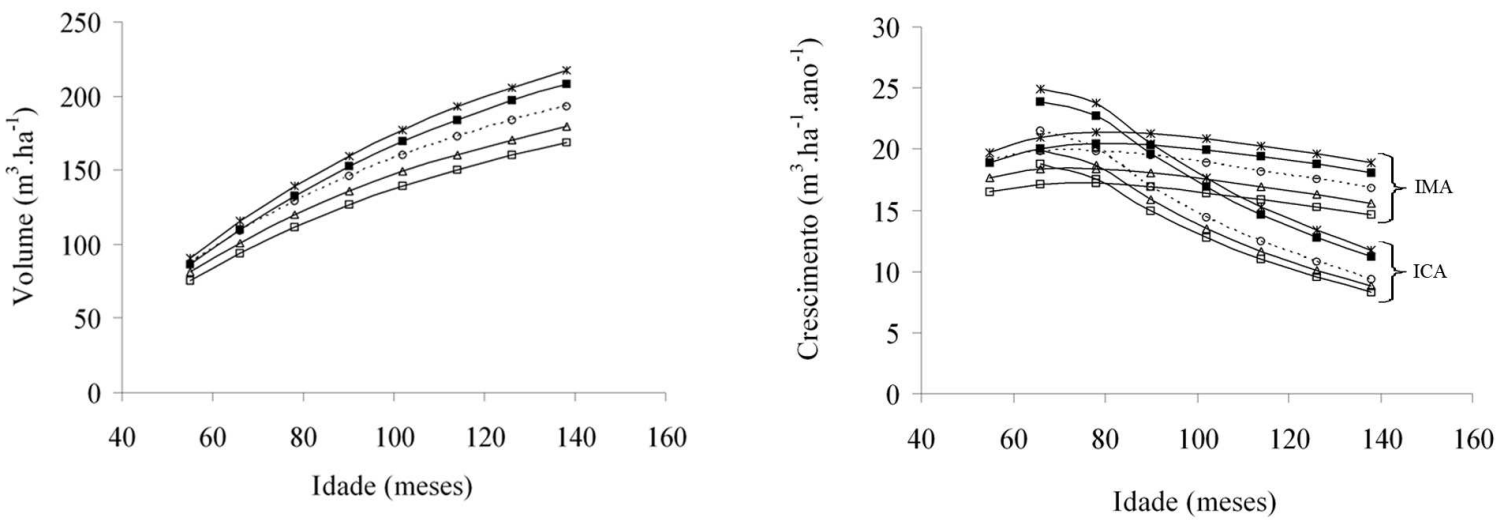

$\longrightarrow 16$ meses $^{1} \longrightarrow-16$ meses $^{2} \cdots 0 \cdots 20$ meses $^{3} \longrightarrow 28$ meses $^{4} \longrightarrow 28$ meses $^{5}$

Figura 3 - Volume $\left(\mathrm{m}^{3} \cdot \mathrm{ha}^{-1}\right)$, incremento médio anual $\left(\mathrm{m}^{3} \cdot \mathrm{ha}^{-1} \cdot\right.$ ano $\left.^{-1}\right)$ e incremento corrente anual $\left(\mathrm{m}^{3} \cdot \mathrm{ha}^{-1}\right.$. ano $\left.{ }^{-1}\right)$ em povoamento do clone 24504 de E. grandis não desbastado, submetido à desrama artificial, em Abaeté, MG. (16, 20 ou 28 meses $=$ idades de primeira intervenção de desrama; 16 meses $^{1}=\mathrm{T} 1, \mathrm{~T} 2$, T3, T5 e T6; 16 meses $^{2}=\mathrm{T} 4 ; 20$ meses $^{3}=\mathrm{T} 1 \mathrm{a}$ T6; 28 meses $^{4}=$ T1 e T7; e 28 meses $^{5}=$ T8 e T9).

Figure 3 - Volume $\left(\mathrm{m}^{3} \cdot h \mathrm{a}^{-1}\right)$, mean annual increment $\left(\mathrm{m}^{3} \cdot h \mathrm{a}^{-1} \cdot y \mathrm{r}^{-1}\right)$ and periodic annual increment $\left(\mathrm{m}^{3} \cdot h \mathrm{a}^{-1} \cdot y r^{-1}\right)$ in a nonthinned stand of $\boldsymbol{E}$. grandis clone submitted to artificial pruning (starting at the age of 16, 20 or 28 months), in Abaeté, MG, Brazil.

enquanto outro grupo foi formado pelos tratamentos T8 e T9, sendo representados pela equação $\operatorname{Ln}(\mathrm{V})=$ 5,71659-72,68841 ( $\left.\mathrm{I}^{-1}\right)$ (Figura 3).

Até os 93 meses de idade, não se observou influência significativa $(\mathrm{p}>0,05)$ da desrama no crescimento das plantas, em todas as variáveis analisadas. Lima (2003) e Polli (2005) já haviam constatado esses resultados em idades mais jovens desse povoamento. Isso decorre da elevada recomposição da copa apresentada pelo material genético estudado (LIMA, 2003). A análise do efeito da desrama deve levar em conta não somente a altura da copa viva da planta, mas também a arquitetura

R. Árvore, Viçosa-MG, v.33, n.5, p.777-787, 2009 
de copa, porque existem materiais genéticos que apresentam elevada concentração de ramos e folhas na base da planta, implicando elevada remoção de área foliar (ALMEIDA, 2003). Pires (2000) verificou que com a remoção de $50 \%$ da altura da copa viva de plantas de Eucalyptus grandis com 1 ano de idade, houve remoção de $81 \%$ da área foliar total. Pinkard (2003) recomendou remoção de até $20 \%$ de área foliar em sítios pobres e até $40 \%$ em sítios mais produtivos. Porém, deve-se levar em conta, também, a capacidade de recuperação e crescimento da copa, o que influencia o posterior crescimento do tronco das plantas (PINKARD, 2002; LIMA, 2003; PULRONIK et al., 2005).

Espera-se, dependendo da intensidade de desbaste, obter maior crescimento das plantas remanescentes. Segundo Leite et al. (2005), após o desbaste as plantas remanescentes apresentam aumento na taxa de crescimento devido à redução de competição entre elas e, consequentemente, aumento na disponibilidade de luz, água e nutrientes proporcionada pelo novo espaçamento entre plantas. Chaves et al. (2007) observaram aumento em $47,38 \%$ na transmitância da radiação fotossinteticamente ativa imediatamente após o desbaste, no mesmo povoamento deste estudo, ou seja, houve favorecimento à entrada de luz no povoamento desbastado, tendo sido observada rápida recomposição da copa até seis meses após o desbaste. Yu et al. (2003) observaram que, em razão da maior penetração da luz no interior do dossel após o desbaste, houve aumento na área foliar e no tamanho dos galhos em Pinus taeda. Porém, o maior crescimento pós-desbaste pode decorrer não apenas devido ao aumento da radiação mas, também, ao aumento na taxa fotossintética. Tschaplinski e Blake (1989) observaram que, após a desbrota em Populus deltoides Bartr. x nigra L. I-262(DN22) e Populus maximowiczii x nigra L. MN9, as folhas dos brotos remanescentes apresentaram taxa fotossintética sete vezes mais elevada do que quando não houve desbrota, indicando que a fotossíntese não estava ocorrendo em toda a sua capacidade. Medhurst e Beadle (2005) observaram aumento na taxa fotossintética após o desbaste, principalmente na base e no meio da copa de Eucalyptus nitens.

Com base nas curvas de ICA e IMA, geradas a partir das curvas de produção, verificou-se que a redução de produtividade deve ocorrer entre 100 e
110 meses de idade no povoamento submetido ao desbaste (Figura 2), indicando haver necessidade de realização do segundo desbaste nessa idade. No povoamento não desbastado, a estagnação ocorreu aos 80 meses (Figura 3). Segundo Kotze e Luckhoff (2007), a intensidade de desbaste deve ser o suficiente para permitir que a árvore tenha crescimento livre por pelo menos um ano, e as novas intervenções de desbaste devem ser realizadas no momento certo, para se obterem madeira na quantidade e tamanho desejados. Segundo esses autores, é importante definir o diâmetro que se pretende obter no final da rotação, bem como o uso dos produtos provenientes do desbaste, para definir as prescrições de desbaste de modo a obter o diâmetro desejado, porém com volume de madeira adequado ao longo da rotação, o que pode ser obtido através de várias intervenções de desbaste.

\section{CONCLUSÕES}

1. A intensidade e frequência de desrama artificial utilizada neste estudo podem ser aplicadas em povoamentos de clone de Eucalyptus grandis com características similares ao deste experimento, sem prejuízo do seu crescimento, devendo-se evitar remoção superior a $60 \%$ da biomassa foliar total, para obter madeira de diâmetro mais elevado.

2. A desrama artificial deve-se iniciar no máximo até 20 meses de idade, no caso do material genético e das condições ambientais deste estudo.

3. Para obter aumento significativo de diâmetro das árvores, no final da rotação o segundo desbaste deve ser realizado próximo às idades indicadas, de 100 a 110 meses.

4. As toras da base das árvores do segundo desbaste, nas maiores classes diamétricas, poderão ser utilizadas para produção de madeira serrada, uma vez que foram desramadas para agregação de valor às toras.

\section{REFERÊNCIAS}

ALMEIDA, M. L. Desrama artificial em clones de híbridos de Eucalyptus grandis x E. urophylla com diferenças em arquitetura de copa. 2003. 119f. Dissertação (Mestrado em Ciência Florestal) - Universidade Federal de Viçosa, Viçosa, MG, 2003.

R. Árvore, Viçosa-MG, v.33, n.5, p.777-787, 2009 
BEADLE, C. et al. Effect of pruning Acacia mangium on growth, form and heart rot. Forest Ecology and Management, v.238, n. 1-3, p.261-267, 2007.

CAMPos, J. C. C.; LEITE, H. G. Mensuração florestal: perguntas e respostas. 2.ed. Viçosa, MG, Universidade Federal de Viçosa, 2006. 407p.

CHAVES, R. A. Dinâmica de copa e crescimento de plantas de clone de Eucalyptus grandis W. Hill ex Maiden submetidas a desrama artificial e a desbaste. 2005. 85f. Dissertação (Mestrado em Ciência Florestal) - Universidade Federal de Viçosa, Viçosa, MG, 2005.

CHAVES, R. A. et al. Dinâmica de cobertura de dossel de povoamentos de clone de Eucalyptus grandis W. Hill ex-Maiden submetidos a desrama artificial e desbaste. Revista Árvore, v.31, n.6, p.989-998, 2007.

DIAS, A. N. et al. Emprego de um modelo de crescimento e produção em povoamentos desbastados de eucalipto. Revista Árvore, v.29, n.5, p.731-739, 2005.

HEIKKILÄ, J.; SIRÉN, M.; ÄIJÄLÄ, O.

Management alternatives of energy wood thinning stands. Biomass and Bioenergy, v.31, n.5, p.255-266, 2007.

KANNINEN, M. et al. Intensity and timing of the first thinning of Tectona grandis plantations in Costa Rica: results of a thinning trial. Forest Ecology and Management, v.203, n.1-3, p.89-99, 2004.

KOTZE, H.; LUCKHOFF, J. A new thinning regime for Eucalyptus grandis sawtimber in Komatiland forests. In: IUFRO 2007 DURBAN - Eucalypts and diversity: Balancing productivity and sustainability, 2007, Durban. Conference Papers. Durban: IUFRO, 2007. 12p. CD ROOM.

LEITE, H. G. et al. Avaliação de um modelo de distribuição diamétrica ajustado para povoamentos de Eucalyptus sp. submetidos a desbaste.

Revista Árvore, v.29, n.2, p.271-280, 2005.

R. Árvore, Viçosa-MG, v.33, n.5, p.777-787, 2009
LELES, P. S. S. et al. Crescimento, produção e alocação de matéria seca de Eucalyptus camaldulensis e E. pellita sob diferentes espaçamentos na região de cerrado, MG. Scientia Forestalis, n.59, p.78-87, 2001.

LIMA, A. P. L. Desrama artificial em clone de Eucalyptus grandis (Hill ex MAIDEN): efeitos sobre o crescimento, a dinâmica de copa e o tempo de desrama. 2003. 195f. Tese (Doutorado em Ciência Florestal) - Universidade Federal de Viçosa, Viçosa, MG, 2003.

MEDHURST, L.; BEADLE, C. L.; NEILSEN, W. A. Early-age and later-age thinning affects growth, dominance, and intraspecific competition in Eucalyptus nitens plantations. Canadian Journal of Forest Research, v.31, n.2, p.187197, 2001.

MEDHURST, J. L.; BEADLE, C. L. Photosynthetic capacity and foliar nitrogen distribution in Eucalyptus nitens is altered by high-intensity thinning. Tree Physiology, v.25, n.8, p.981-991, 2005.

NOGUEIRA, G. S. et al. Determinação da idade técnica de desbaste em plantações de eucalipto, utilizando o método dos ingressos percentuais. Scientia Forestalis, n.59, p.51-59, 2001.

NOGUEIRA, G. S. et al. Modelo de distribuição diamétrica para povoamentos de Eucalyptus sp. submetidos a desbaste. Revista Árvore, v.29, n.4, p.579-589, 2005.

PEREIRA, J. C. D. et al. Características da madeira de algumas espécies de eucalipto plantadas no Brasil. Colombo: Embrapa Florestas, 2000. 113p. (Embrapa Florestas. Documentos, 38).

PINKARD, E. A. Effects of pattern and severity of pruning on growth and branch development of precanopy closure Eucalyptus nitens. Forest

Ecology and Management, v.157, n.1-3, p.217230, 2002.

PINKARD, E. A. Physiological and growth responses related to pattern and severity of green pruning in young Eucalyptus globulus. Forest Ecology and Management, v.182, n.1-3, p.231-245, 2003. 
PINKARD, E. A. et al. Growth responses, physiology and decay associated with pruning plantation-grown Eucalyptus globulus Labill. and E. nitens (Deane and Maiden) Maiden. Forest Ecology and Management, v.200, n.1-3, p.263-277, 2004.

PIRES, B. M. Efeito da desrama artificial no crescimento e qualidade da madeira de Eucalyptus grandis para serraria e fabricação de móveis. 2000. 96f. Dissertação (Mestrado em Ciência Florestal) - Universidade Federal de Viçosa, Viçosa, MG, 2000.

PIRES, B. M.; REIS, M. G. F.; REIS, G. G. Crescimento de Eucalyptus grandis submetido a diferentes intensidades de desrama artificial na região de Dionísio, MG. Brasil Florestal, v.21, n.73, p.14-22, 2002.

POLLI, H. Q. Crescimento e qualidade da madeira para serraria em clone de Eucalyptus grandis [Hill ex Maiden] submetido à desrama artificial. 2005. 97f. Dissertação (Mestrado em Ciência Florestal) Universidade Federal de Viçosa, Viçosa, MG, 2005.

POLLI, H. Q. et al. Qualidade da madeira em clone de Eucalyptus grandis Hill ex Maiden submetido a desrama artificial. Revista Árvore, v.30, n.4, p.557$566,2006$.

PULROLNIK, K. et al. Crescimento de plantas de clone de Eucalyptus grandis [Hill ex maiden] submetidas a diferentes tratamentos de desrama artificial, na região de cerrado. Revista Árvore, v.29, n.1-3, p.495-505, 2005.
SHUMACHER, F.X.; HALL, F.S. Logarithmic expression of timber-tree volume. Journal of Agricultural Research, v.47, n.9, p.719-734, 1933.

SULLIVAN, T. P. et al. Long-term responses of ecosystem components to stand thinning in young lodgepole pine forest III. Growth of crop trees and coniferous stand structure. Forest Ecology and Management, v.228, n.1-3, p.69-81, 2006.

TREVISAN, R. et al. Efeito da intensidade de desbaste nas características dendrométricas e tecnológicas da madeira de Eucalyptus grandis. Ciência Florestal, v.17, n.2, p.377-387, 2007.

TSCHAPLINSKI, J. T.; BLAKE, T. J.

Photosynthetic revigoration of leaves following shoot decapitation and accelerated growth of coppice shoots. Physiologia Plantarum, v.75, n.2, p.157-165, 1989.

VALE, R. S. et al. Efeito da desrama artificial na qualidade da madeira de clones de eucalipto, em sistema agrossilvipastoril. Revista Árvore, v.26, n.3, p.285-297, 2002.

VALINGER, E.; ELFVING, B.; MORLING,T.Twelve-year growth response of Scots pine to thinning and nitrogen fertilization. Forest Ecology and Management, v.134, n.1-3, p.45-53, 2000.

YU, S. et al. Crown characteristics of juvenile loblolly pine 6 years after application of thinning and fertilization. Forest Ecology and Management, v.180, n.1-3, p.345-352, 2003. 